\title{
The influence of brush cutter's vibrations on human body
}

\author{
Aurica TRUŢA ${ }^{1, \text { a }}$, Felicia Aurora CRISTEA ${ }^{2, b}$ and Mariana ARGHIR ${ }^{3, c}$ \\ 1,2,3 B-dul Muncii 103-105, Cluj-Napoca, Romania \\ aauricatruta@yahoo.com (corresponding author), bcristea_fa@yahoo.de, \\ cmarianaarghir@yahoo.com
}

Keywords: human body vibration, brush cutter, transmitted vibration, measurement

\begin{abstract}
In recent years there have been many cases of HAVS being reported for people who work in agriculture, horticulture, landscape gardening and forestry. The work described in this paper assesses the transmitted vibration to the human body from a hand guided power tool, means a brushcutter. As it is known the brushcutters induce a high level of vibrations on human operators. Depending by brushcutter's type, exposure time and human subjectivity, we can discuss about the vibration influence on human body. Long exposure to hand-arm vibration, whole body vibration and mechanical overloading is considered a potential cause of professional diseases.
\end{abstract}

\section{Introduction}

The relation between vibrations and back pain has been studied since 1950s [13]. Several studies have shown that prolonged and excessive whole body vibration (WBV) or hand-arm vibration (HAV) is considered as a general physical stressor and has been associated with a variety of occupational health disorders especially of pains in the hands and lower arm, back pain and herniated lumbar disc problems [1,4,5].

Farmers are a group of workers often exposed to mechanical shocks, HAV and WBV. Hand held mechanical tools and awkward posture are low back pain risk factors [9].

In a farm the most important sources of mechanical vibrations are the electro-mechanical tools. In it's daily activity a farmer can use one or more vibrational tools like handheld power tools, or powered machines which process handheld materials [10]. Here it can be mentioned: brushcutter, chainsaw, electric saw, wood planing machine.

Vibration emissions of brushcutter are sufficient to present an occupational risk of vibration injury. The body will take an amount of vibrational wave which it transmits to its entire structure. The human body can behave either as a vibration damper or as an amplifier. Its mechanical properties can be easily modified so frequent exposure to HAV or WBV can lead to permanent health effects.

The human locomotor apparatus is very important for the body movement. Vertebral spine is the central structural element that supports the upper body and shelters the largest bus nerve, so that frequently the disorders and spinal deformities have neurological consequences.

Spine disorders can be the results of vibration exposures combined with other factors. Working posture, anthropometric characteristics, physical workload, noise and individual susceptibility (age, pre-existing disorders) can bring its contribution on spine disorders.

Intervertebral disks function mechanically to support high compressive loads and shear deformations while permitting rotational flexibility [7]. The intervertebral disc (IVD) is prone to ruptures and degenerative process. Mechanical stress applied to the disc appears to accelerate the development of degenerative changes [12].

The objective of this paper is to investigate the vibration transmissibility from a hand held and hand guided tool used by a farmer. We consider a middle farm, or a farm where there is no possibility to use a sickle mower which is a machine for cutting grass or other plants that grow on the ground, where the farmer need to use a brushcutter. 
Materials and methods

Brushcutter's technical caracteristics.

The brushcutter has equivalent vibration level (ahv, eq) left / right handle $2.5 / 2.4 \mathrm{~m} / \mathrm{s}^{2}$ (emission) (fig. 1). It's engine power is $1,6 \mathrm{KW}$, maximum power speed: $11500 \mathrm{rpm}$, engine capacity is $34.6 \mathrm{~cm}^{2}$, sound pressure level at operators ear: $96 \mathrm{~dB}(\mathrm{~A})$, noise emissions: $113 \mathrm{~dB}(\mathrm{~A})$, weight (without cutting equipment): $6.2 \mathrm{Kg}$, tube length: $1483 \mathrm{~mm}$.

Other technical characteristics:

Diameter of cylinder $38 \mathrm{~mm}$

Fuel consumption $480 \mathrm{~g} / \mathrm{kWh}$

Idling speed $2900 \mathrm{rpm}$

Level of emissions (CO medium) $196 \mathrm{~g} / \mathrm{kWh}$

Level of emissions (HC medium) $32 \mathrm{~g} / \mathrm{kWh}$

Level of emissions (NOx medium) $2 \mathrm{~g} / \mathrm{kWh}$

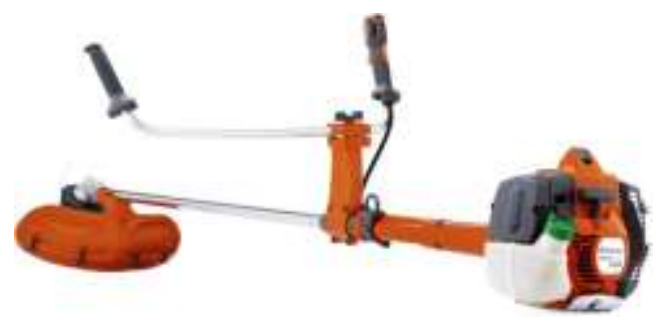

Figure 1. Brushcutter.

Participants. Experimental method consists in measurements on a healthy person, a man, able to develop its daily activity. He was observed for three years consecutively. The subject was about 90 $\mathrm{kg}, 180 \mathrm{~cm}$ tall, and 42-44 years old, healthy from medical point of view. Both hands of farmer are in contact with the brushcutter. More than that, the brushcutter has another contact with the human body on the right feet. The subject used anti-vibration gloves (Fig. 2).
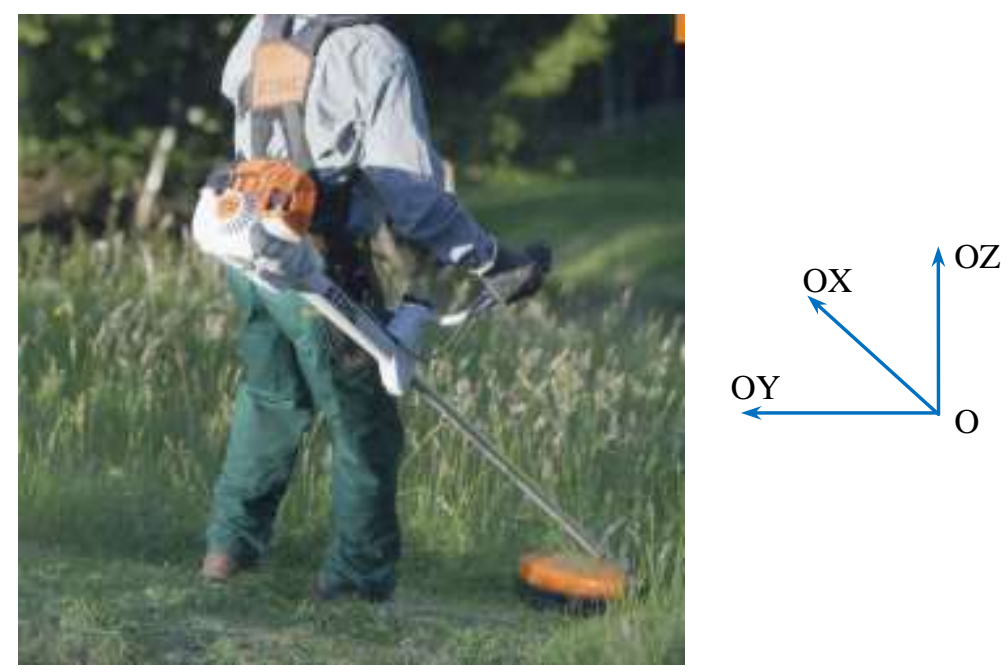

Figure 2. Farmer using a brushcutter.

Hand arm vibration. The exposure limit value (ELV) is the maximum amount of vibration which an operator may be exposed to on any single day. For Hand-Arm Vibration, ELV is a daily exposure of $5 \mathrm{~m} / \mathrm{s}^{2} \mathrm{~A}(8)$. The Exposure Action Value is $2.5 \mathrm{~m} / \mathrm{s}^{2} \mathrm{~A}(8)$ [11]. It represents a high risk above which workers should not be exposed. Workers should use anti-vibration gloves to protect themselves against HAV. The hand guided powered equipment, has its own protection to vibration transmissibility, but they cannot completely block the vibration to acts on human arms. Long exposure to HAV is associated with White Finger Syndrome (WFS) or Raynaud's Syndrome. 
Whole body vibration. When mechanical vibration acts on feet or on other parts of the body, they are called Whole Body Vibration. Long exposure to WBV is strong associated with low back pain [14]. For Whole Body Vibration, ELV is a daily exposure of $1.15 \mathrm{~m} / \mathrm{s}^{2} \mathrm{~A}(8)$ and the EAV is $0.5 \mathrm{~m} / \mathrm{s}^{2} \mathrm{~A}(8)$ [11]. There is no available any kind of protection equipment against WBV.

'On-farm' WBV and HAV exposure measurement. The brushcutter is an ustensille used by a farmer a few times in one year. If he use that for five times in one year and have to cut the grass from $5000 \mathrm{~m}^{2}$ that means he use the brushcutter for 8 hours along of 5 days, means 40 hours exposure time.

All measurements were performed with vibration analyzer SVAN958. It was used a three dimensional accelerometer attached in different places on the human body. Contact body with the accelerometer it was provided by an elastic band (fig.4).

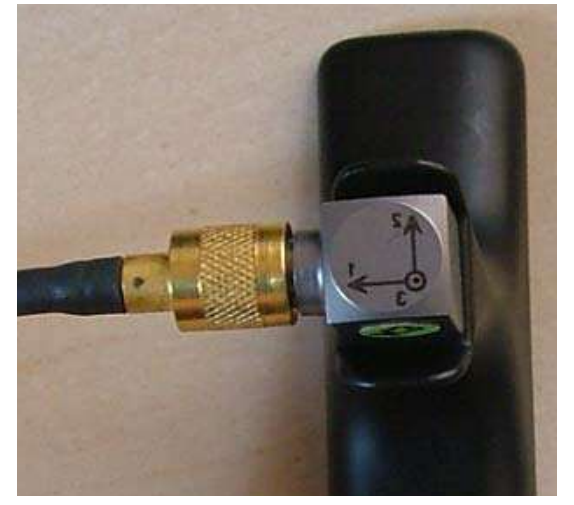

Fig. 3. The three dimensional used accelerometer
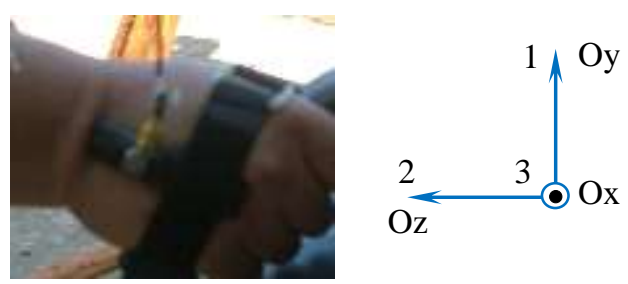

Figure 4. Elastic band sustain the accelerometer

The vibration axis were considered like in STAS ISO 8727/2001for WBV: The positive OZ axis is from the feet to the head, along the body, the pozitive OY axis is from the right to the left and the pozitive OX axis is from the back to the front (Fig. 2), and STAS ISO 5349 for HAV (Fig. 4). The weighted filter used is Wk for standing person and Wh for hands (STAS ISO 2631/2001).

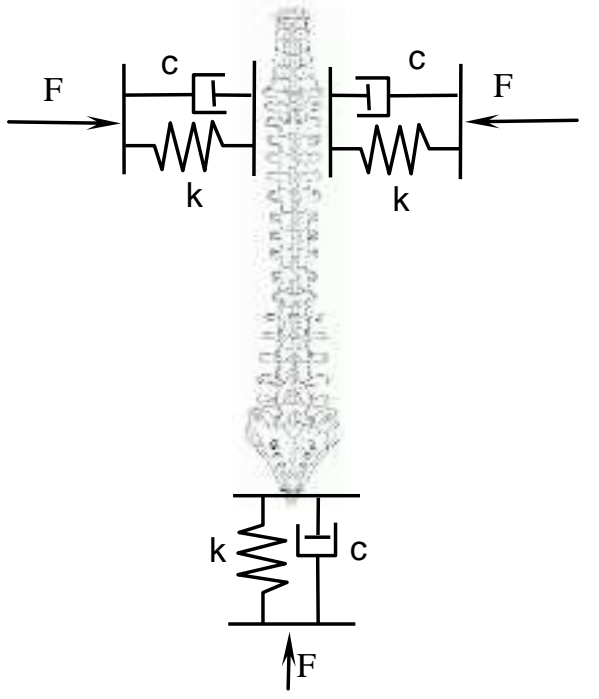

Figure 5. Simplified mechanical model for vibration acting on human spine from hand-arm and feet directions

Brushcutter is sustained by human operator on his shoulders and in the same time on his right feet. He controls it with the hands. Human operators position for the trimming operation is standing and little bended forward. Human spine is exposed to vibrational wave from two directions: from shoulders direction and from the right feet direction (fig. 5). The brushcutter's weight about $6.2 \mathrm{Kg}$ provide a supplemented force that load the human spine and IVD. The outside temperature was between $24^{\circ} \mathrm{C}$ and $29^{\circ} \mathrm{C}$. These were the full and real conditions.

The measurements were performed only on human body and in full load conditions. It was a real farmer that usually performs this job. The vibration analyzer do not created discomfort for the subject. First, the accelerometer was attached on the right hand. Its contact with the body was created between a special supports, right on the skin. An elastic band kept the accelerometer and its support in unmovable position. The $\mathrm{Oz}$ axis is considered along of the hand-arm. The position of accelerometer is like in figure 4. 
The other measurements were on the left hand, on the right feet, on the lumbar spine, on the right shoulder and on the left shoulder. The vibration level on the left handle was higher that on the right handle.

Figure 6 presents the contact points with the excitation forces. There are three of them right on the tool and two of them are on the left shoulder and right shoulder that sustain the brushcutter's harness. Point 1 represents the contact between left hand and left handle. Point 3 represent the contact between right hand and right handle. There is a strong contact between the tool and right feet in point 2. Points 3 and 4 are the area where the body sustains the harness: shoulders girdle.
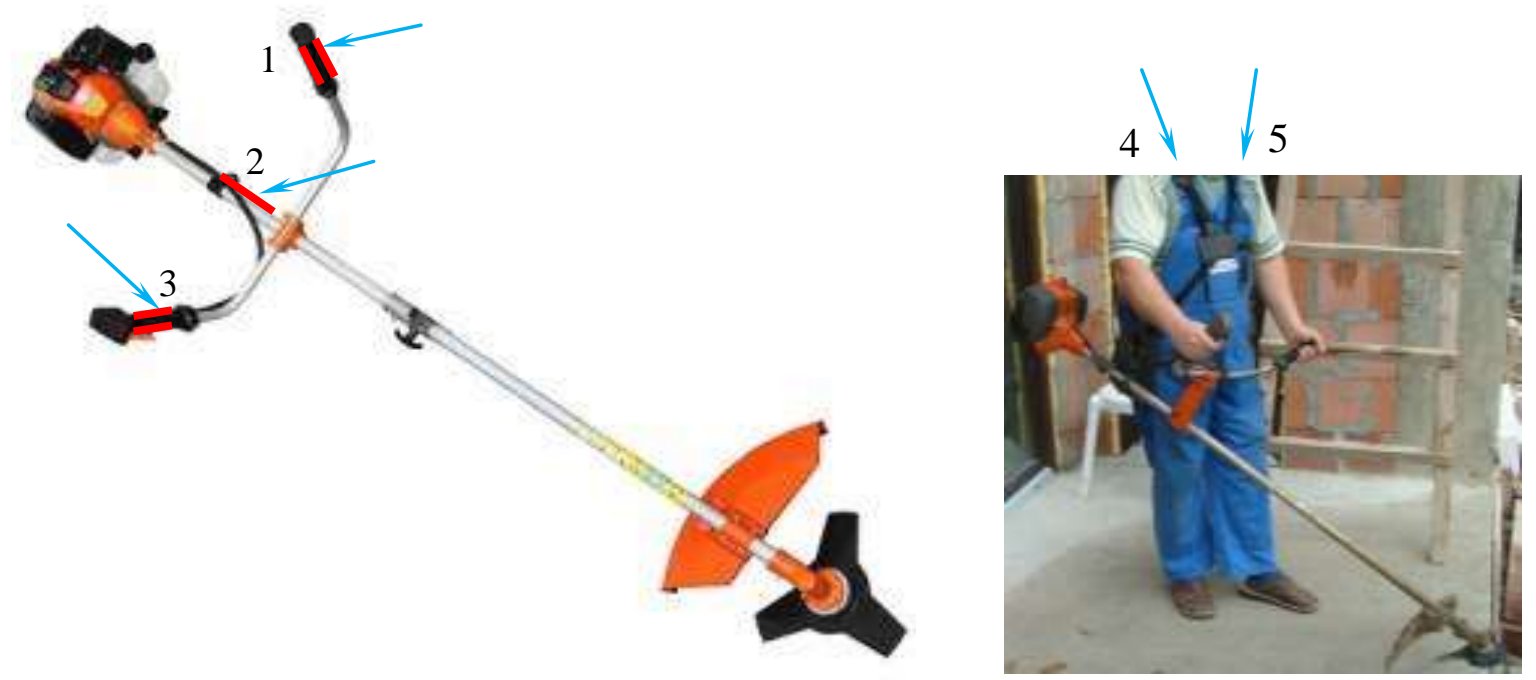

Figure 6. Brushcutter position on the human body.

\section{Results and discussion}

Measurements working with brushcutter were performed on the right hand, left hand, right feet, left and right shoulder and lumbar spine. They were performed in the same activity but not in the same time.

For the right wrist the higher values were on OY axis, means lateral motion: right to the left side and left to the right side. The top RMS acceleration value was $2451.884 \mathrm{~mm} / \mathrm{s}^{2}$ (Fig. 7).

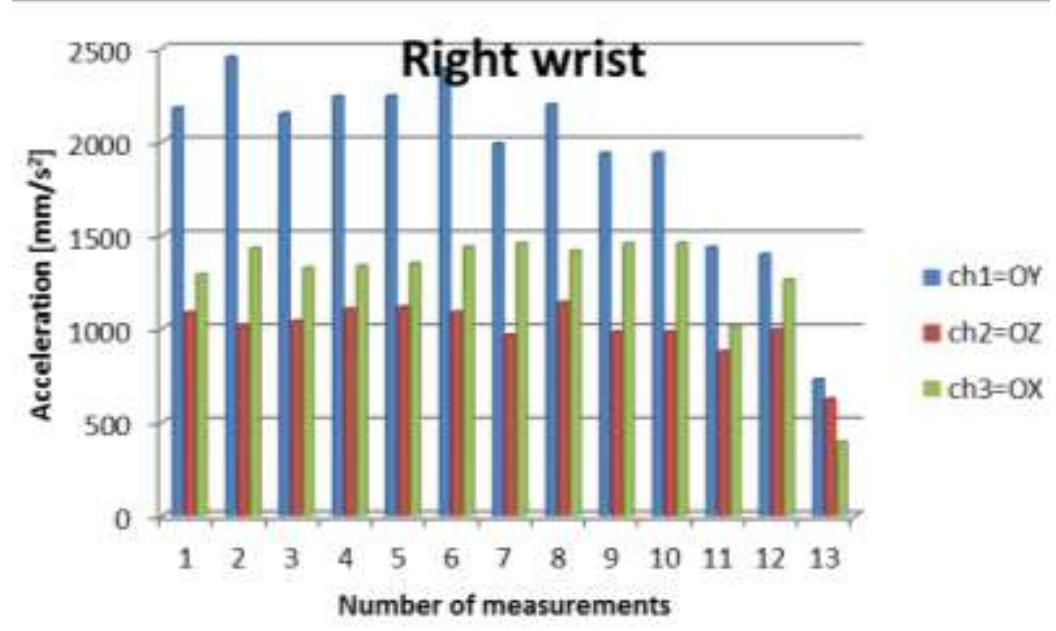

Figure 7. RMS acceleration measured on the right wrist. 
For the left wrist the highest value is much smaller than that of the right hand. This time there is a top of value on OX axis, means from back to front side (Fig. 8).

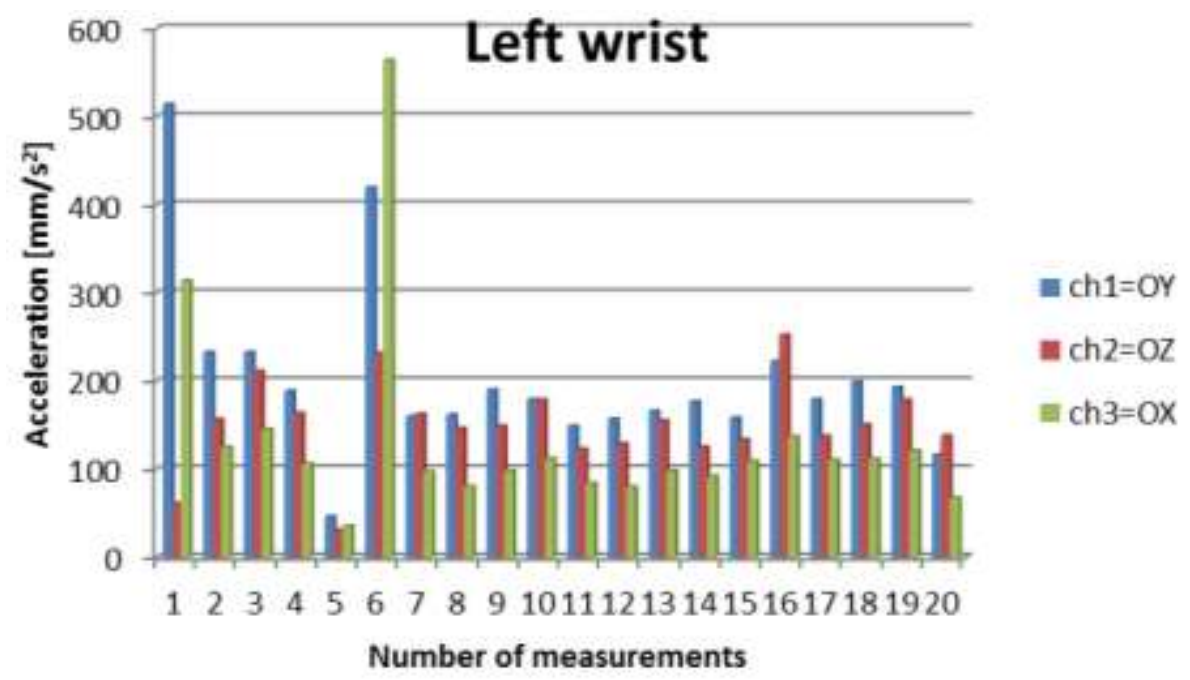

Figure 8. RMS acceleration measured on the left wrist.

There is a big difference between the left and right hand values. It is own to different moments of measurements (Fig. 9). Means that it is important to have measurements, for different places on the human body, in the same time for the same operation.

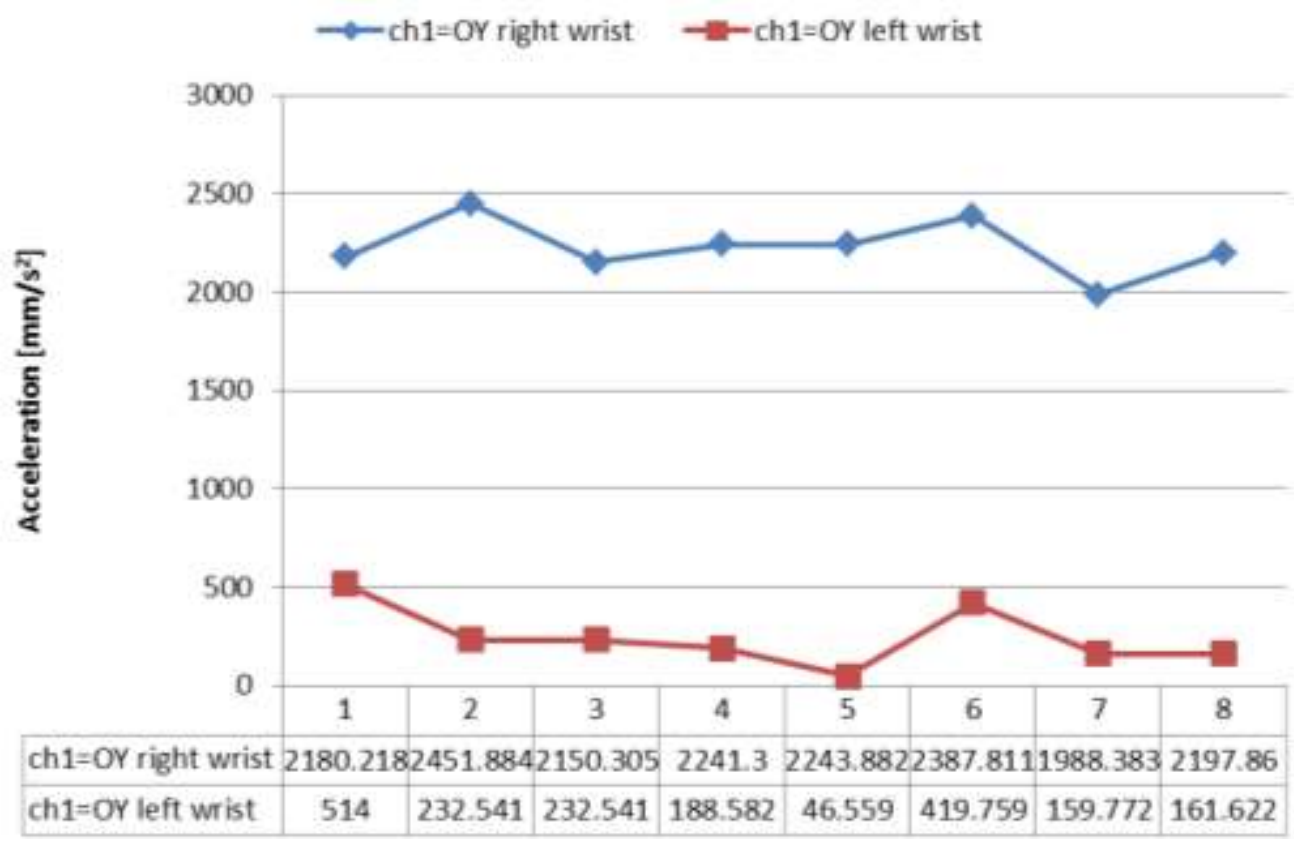

Figure 9. Comparison between left and right wrist

On the right feet there are some values comparable with right wrist. The contact with the human body is so strong in both places. The top value on the right feet is about $3069 \mathrm{~mm} / \mathrm{s}^{2}$ (Fig. 10). It is higher than the top value for the right wrist (Fig. 11).

The transmitted vibrations to the lumbar spine are to big (Fig. 12) (Table 1). The motion is transmitted from five points. Overlapping vibrations of the five points of contact results in big values measured on the lumbar spine. Such high values, applied for a long time, lead initially to discomfort that can degenerate into serious diseases. 


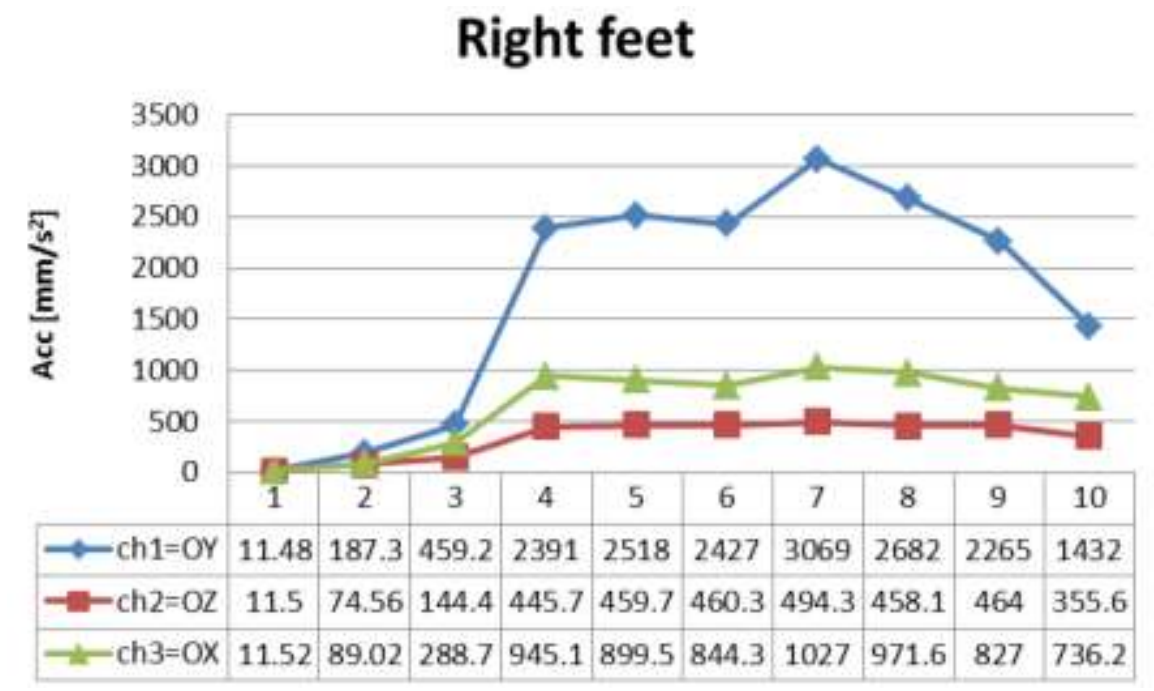

Figure 10. RMS acceleration measured on the right feet.

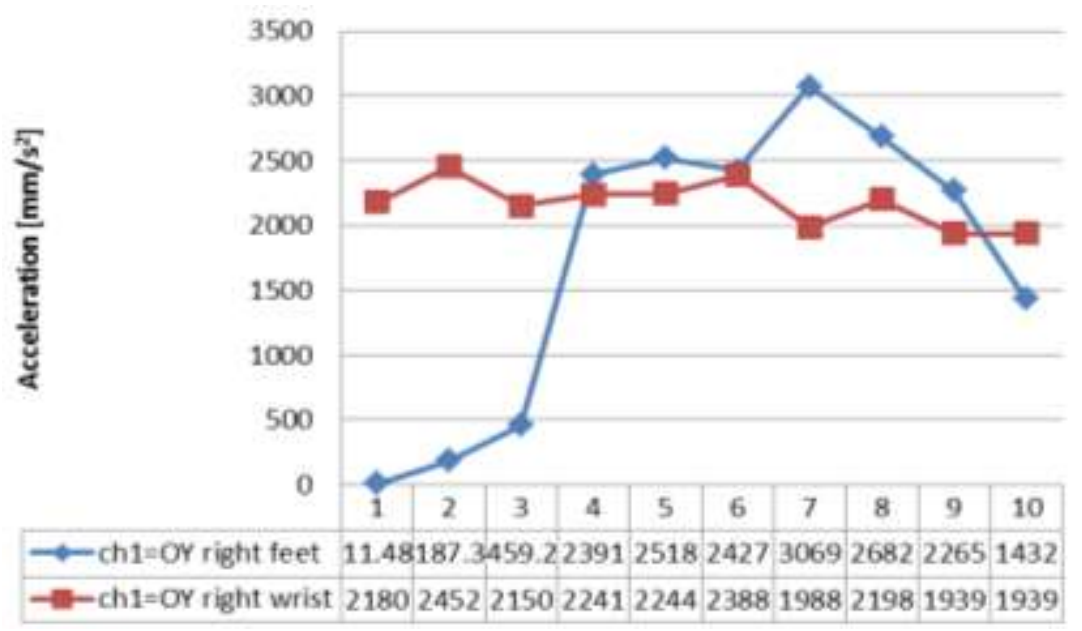

Figure 11. Comparison between RMS acceleration measured on the right feet and RMS acceleration measured on the right wrist.

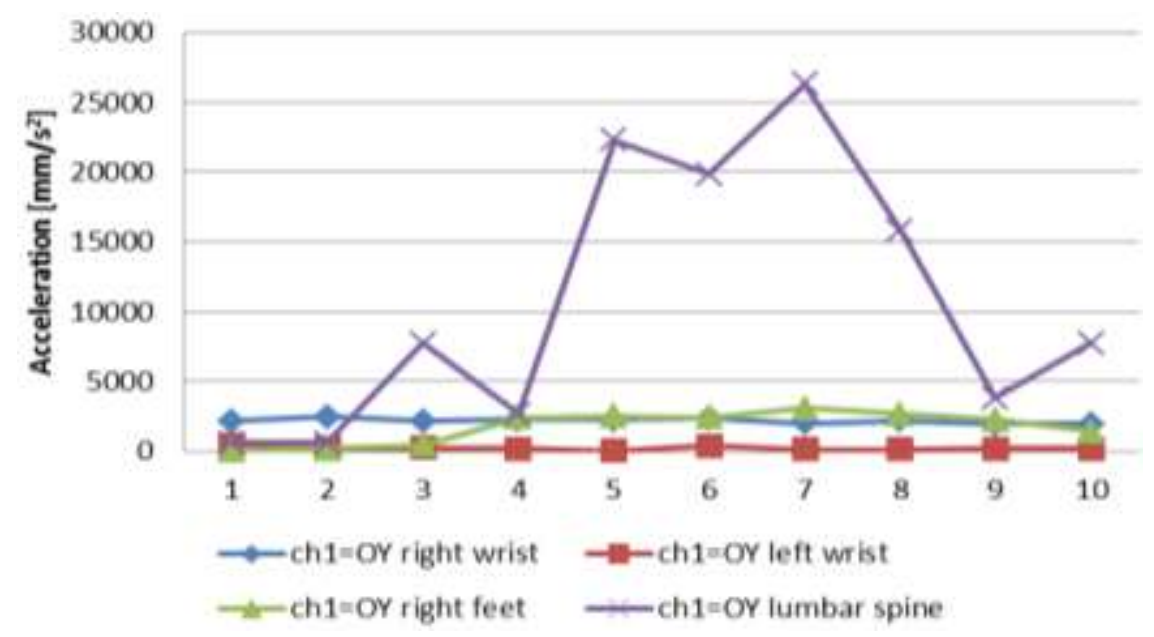

Figure 12. Transmitted vibration from a brushcutter to left and right wrist, to right feet and to lumbar spine. 
Table 1. Measured vibration on human body working with a brushcutter

\begin{tabular}{|r|r|r|r|r|r|r|r|r|}
\hline right wrist & 2150.305 & 2241.3 & 2243.882 & 2387.811 & 1988.383 & 2197.86 & 1938.653 & 1938.653 \\
\hline left wrist & 232.541 & 188.582 & 46.559 & 419.759 & 159.772 & 161.622 & 190.327 & 179.267 \\
\hline right feet & 459.198 & 2390.562 & 2517.677 & 2426.61 & 3069.022 & 2682.255 & 2264.644 & 1432 \\
\hline lumbar spine & 7745 & 2669.931 & 22259 & 19861 & 26333 & 15831 & 3819 & 7718 \\
\hline
\end{tabular}

\section{Conclusion}

The body position, and its angle with the vertical direction, it was not taken in consideration in this study, neither in vibration transmissibility thru human body.

Hands are both exposed to vibration. There is a difference between the measured vibration on right hand and measured vibration on left hand. The values for right hand are higher than similar values on left hand. This is a normal situation because right hand has principal duty and it is closer by engine that the other hands. That means that it is possible to have some amplified vibration thru the handle or this values can become from different moment of measurements.

When the subject uses the brushcutter, his right foot is a support for the hand held tool. The position of brushcutter's harness is on the farmer's shoulders. So the tool will transmit the emission vibration from three different directions: from hand-arms, from his right feet and from his shoulders.

The problem is that the vibration acts on the five points in the same time. So the vibration wave on vertebral lumbar spine are combined (Fig. 6). Prolonged exposure to these values of vibration has a strong impact on a human subject. While vibration values recorded at hands were framed in the permissible values, the spine measured values were above permissible limits. High levels and long exposure can cause intervertebral disc herniation among others.

One big disadvantage for this study is that the measurement has not been done in the same moment for each part of the body. It was necessary to stop the tool and move the accelerometer for each episode of measurements. In these situations there are possible differences between tools accelerations and its vibration emission. It can be differences even between work conditions: grass can have different orientations or dimensions and can be higher or less than before.

Anyway operator accuses back pain and hand pain after three hours of work. Also feel tingling in hands and right leg. Vibration emissions of brushcutters are sufficient to present an occupational risk of vibration.

Damping existing solutions are not enough. Gloves are good absorbers but not enough to eliminate the risk of disease or injury. Point is that the contact between the foot and the tool should be minimized. Need to be found solutions to minimize the transmissibility from handle to hands.

\section{Acknowledgment}

This paper was supported by the project "Development and support of multidisciplinary postdoctoral programmes in major technical areas of national strategy of Research - Development Innovation" 4D-POSTDOC, contract no. POSDRU/89/1.5/S/52603, project co-funded by the European Social Fund through Sectoral Operational Programme Human Resources Development 2007-2013. 


\section{References}

[1] B. Bazrgaria, A. Shirazi-Adla,,, M. Kasra, Computation of trunk muscle forces, spinal loads and stability in whole-body vibration, Journal of Sound and Vibration 318 (2008), pp. 13341347

[2] Samantha C.W. Chan, Stephen J. Ferguson, Benjamin Gantenbein-Ritter, The effects of dynamic loading on the intervertebral disc, European Journal Spine (2011) 20: 1796-1812, DOI 10.1007/s00586-011-1827-1

[3] Health and Safety Laboratory, Hand-arm vibration of horticultural machinery, 2011, http://www.hse.gov.uk/research/rrpdf/rr894.pdf

[4] Ingo Hermanns, Nastaran Raffler, Rolf P. Ellegast, Siegfried Fischer, Benno Gores, Simultaneous field measuring method of vibration and body posture for assessment of seated occupational driving tasks, International Journals of Industrial Ergonomics 38 (2008), pp. 255-263.

[5] I. Hermans, N. Raffler, R.P. Ellegast, S. Fischer, B. Gores, Simultaneous field measuring method of vibration and body posture for assessment of seated occupational driving tasks, International Journal of Industrial Ergonomics 38, pag. 255-263, 2008

[6] James C Iatridis, Iolo ap Gwynn, Mechanisms for mechanical damage in the intervertebral disc annulus fibrosus, Journal of biomechanics 37 (2004) pp 1165-1175.

[7] James C Iatridis, Masaru Furukava, Ian A.F. Stokes, Mack G. Gardner-Morses, JeffreyP. Laible, Spatially resolved streaming potentials of human intervertebral disk motion segment under dynamic axial compression, Journal of Biomechanical Engineering, 2009, 131(3): 031006.doi:10.1115/1.3005164

[8] M.Y. Lu, C.W. Hutton, M.V. Gharpuray, Can variations in intervertebral disc height affect the mechanical function of the disc?, Spine, 21 (1996), pp. 2208-2217

[9] Stephan Milosavljevic, Frida Bergman, Borje Rehn, Allan B. Carman, All-terrain vehicle use in agriculture: Exposure to whole body vibration and mechanical shock, Applied Ergonomics 41 (2010), pp. 530-535

[10] [Net 01] Control the risks from hand-arm vibration. Advice for employers on the Control of Vibration at Work. Regulations 2005. http://www.hse.gov.uk/vibration/hav/statistics.htm

[11] [Net02]http://www.healthyworkinglives.com/advice/workplace-hazards/vibration.aspx

[12] Ngwa M., A mathematical model of the compression of a spinal disc, Mathematical Biosciences and Engineering, Vol. 8, Nr.4, 2011, pp.1061-1083

[13] Taryn E. Hill, Geoffrey T. Desmoulin, Christopher J. Hunter, Is vibration truly an injurious stimulus in the human spine?, Journal of Biomechanics 42 (2009), pp. 2631-2635

[14] Mechanical vibration. Guide to the health of vibration on the human body. 